\title{
Assessment of Knowledge and Practice on Consumption of Expired Pharmaceuticals: A Cross-Sectional Study at Karachi, Pakistan
}

\author{
Kiran Rafiq ${ }^{1}$, Muhammad Bilal Azmi ${ }^{2 *}$, Zafar Saied Saify ${ }^{3}$, Waqasuddin Khan $^{4}$, Irfan Ashraf ${ }^{1}$, Najia Rahim ${ }^{5}$, Greesh \\ Kumar $^{1}$, Naureen Baloch ${ }^{1}$ \\ ${ }^{1}$ Jinnah Sindh Medical University, Karachi, Pakistan. ${ }^{2}$ Quality Enhancement Cell, Dow University of Health Sciences, Karachi, Pakistan. ${ }^{3}$ H.E.J. Research \\ Institute of Chemistry, International Centre for Chemical \& Biological Sciences, University of Karachi, Karachi Pakistan. ${ }^{4}$ Jamil-ur-Rahman Center for \\ Genome Research, International Center for Chemical and Biological Sciences, University of Karachi, Karachi, Pakistan. ${ }^{5}$ Dow College of Pharmacy, Dow \\ University of Health Sciences, Karachi, Pakistan.
}

\section{ARTICLE INFO}

Article history:

Received on: 29/01/2015

Revised on: 18/02/2015

Accepted on: 26/02/2015

Available online: 27/04/2015

Key words:

Data, frequency, self-

administered, medicines.

\begin{abstract}
The objective is to assess the awareness level and practice for the consumption of expired pharmaceuticals in general public of Karachi, Pakistan. In this regard, a cross-sectional study was conducted and completed from December 2013 to September 2014. Self-administered study instrument was used which consisted of total 11 variables regarding the assessment of participants' perspective on product expiry and its usage was distributed to 120 participants. Data were analyzed in SPSS version16.0. Primarily descriptive nature was used while frequency percentages of respondents were computed for categorical variables. Mean \pm SD were computed for assessment of knowledge scores. Overall response rate was $83.33 \%$ in which 74 male and 26 females filled the study instrument. Average scores of respondents' awareness regarding expiry of pharmaceutical products was $4.62 \pm$ 0.75. The qualification of respondents includes the graduates' count (64.9\% male and $65.4 \%$ females) while $16.2 \%$ male and $23.1 \%$ female were postgraduate. Response distribution of respondents showed that $93.2 \%$ male and $92.3 \%$ female were aware about the product expiry concept. Similarly, $87.8 \%$ male and $96.2 \%$ female were in favor for their non-utilization, as $89.2 \%$ male and $96.2 \%$ female reported its severe harmful effects. Overall, $87.8 \%$ male and $76.9 \%$ female were interested to discard such products. Interestingly, $82.4 \%$ male, $73.1 \%$ female were in full opinion to check the product expiry date before its purchase whereas, $78.4 \%$ male and $92.3 \%$ female have their opinion to purchase long expiry products. Box plot regarding products preference and total aggregated knowledge scores showed consistency. Results concluded that the knowledge, responses and practices of participants were in favor for the non-utilization of expired pharmaceuticals.
\end{abstract}

\section{INTRODUCTION}

The date of expiration of any pharmaceutical product is considered as the final day of its utilization in terms of medication (Persson et al., 2009). The expiry date for pharmaceuticals is the point in time when a product is no longer within an acceptable condition to be considered effective as well as safe for health of consumers (Al-Gedadi et al., 2008; Shah et al., 2011). Depending on the product, the expiry date may be set as a fixed time after

\footnotetext{
* Corresponding Author

Dr. Muhammad Bilal Azmi, Quality Enhancement Cell, Dow University of Health Sciences, Karachi, Pakistan.Email: azmibilal@gmail.com
}

manufacturing or after dispensing or after opening of the manufacturer's container (Ali et al., 2010). However, it is not a fix concept that all drugs deteriorate at the same rate or interval of time (Lyon et al., 2006) whereas, medicines belong to the different synthetic or formulated categories marks the shelf life position (Kona et al., 2014). According to the World Health Organization, pharmaceutical products have a defined package insert which gives all the essential information regarding the usage of medicines, undesirable effects, interactions and the expiry date (Abdo-Rabbo et al., 2009). The Food and Drug Administration (FDA) since 1979, has made obligatory for pharmaceutical manufacturers to mention expiration dates on every pharmaceutical products (Swaroop and Varun, 2011). 
In this regard, the majority of medicines sold in the America, expiration dates are clearly provided which usually range from 12 to 60 months from the date they are manufactured (Swaroop and Varun, 2011). The terms 'expiry date' or 'shelf-life' or 'best before' is applied to products like beverages, food items, pharmaceutical products, chemicals, cosmetics etc (Singh, 1999). In most of the cases of pharmaceutical products, the useful life of product or its expiry date reflects the product quality as after the expiration, contamination is observed by increased levels of microbial index or moisture contents can also influence the nonutilization of such medicines (Bajaj et al., 2012).

It has been well reported that due to the lack of interest the consumer is not attempted to focus on the expiry or short expiry date of product, and easily purchase such products. However, due to non-awareness if expired product is used, its therapeutic effectiveness decreases over time or it may have some undesirable impacts on human health (Carstensen, 2000; Swaroop and Varun, 2011). Earlier, it was also reported that some medications produces the risk of lethality very after their expiry date, as the product composition starts to decomposed into its constituents (Shah et al., 2011; Asti et al., 2012). Additionally, the situation becomes more dangerous when a person has some emergency and without awareness of product expiry what they actually purchased in term of medication. Therefore, there is always a need that reflects the complete awareness of product expiration concept before purchase or use. Hence, present study was undertaken to explore the knowledge, practice and consumption of expired pharmaceuticals among general public of Karachi, Pakistan.

\section{STUDY METHODS}

Study design of present research survey was crosssectional and non-experimental. The data were collected from general public of Karachi, Pakistan. Participants were chosen irrespective of their occupational affiliations, age, marital status, academic qualification and race. Present study was completed in duration from December 2013 to September 2014. Sample size of study participant represents undergraduate, graduates and postgraduates of Karachi.

\section{Ethics statement}

Before handing over the survey instrument, informed verbal consent was obtained from studied participants. At the same time, assurance of confidentiality of all their shared information as well as professional affiliation was given. In every step of this survey, the identity of participants remains anonymous.

\section{Sample size}

Sample size was calculated through online Raosoft sample size calculator. Sample size was computed on the basis of adjusted the margin of error (d) at $10 \%$ and confidence of interval on $95 \%$, with the recommended population size of 2000 and at least $50 \%$ response distribution the minimum sample size was equal to 96 participants as minimum target. Adding $20 \%$ nonresponse rate, the sample size was augmented upto 115 respondents.

\section{Study Instrument}

All subjects were assessed for the socio-demographic characteristics like age, gender, experience, qualification and marital status. The research questionnaire consists of total eleven (11) variables/ questions, in which question 1 to 7 has scaling of value one (1) or zero (0). Responses to question 1 to 7 were equally considered as YES or NO. For the convenience of respondents, at the end of questionnaire it was also prescribed that all YES marks was scored 1 (one) while all NO options were designated as zero (0) score. However, question 8 to 11 represents the expression of choice, and preference of respondents which actually reflects the usage or discard of expired products from the selected sample size. After completion of each questionnaire the total aggregated score of every participant was computed from question 1 to 7 only.

The scale to assess the knowledge regarding the usage or discard of expired product was also provided with this questionnaire as; a) Score 0 to 2.5 showed minimum level of awareness regarding the usage of expired pharmaceutical products: b) Score 2.6 to 4.5 showed some average level of awareness regarding the usage of expired pharmaceutical products: c) Score 4.6 to 7 showed highest level of awareness regarding the usage of expired pharmaceutical products.

\section{Participants' Enrolment}

Total 120 individuals were approached to fill the present research survey. One hundred (100) individual only filled the complete questionnaire. Thirteen (13) individuals had regretted there availability while seven (07) participants did not report their response completely/ properly on provided instrument. Overall twenty (20) individuals were excluded as study participants. Therefore, an approximate of $83.33 \%$ was the total rate of return of present study questionnaire.

\section{Data Analysis}

Data were entered and analyzed in Statistical Package for Social Sciences (SPSS version16.0) software for Windows version 7 professional. Frequencies and percentages of respondents were computed for categorical variables like age, gender, academic qualification, marital status, and years of experience. Mean \pm Standard Deviation (SD) were computed for assessment of knowledge regarding the usage or discard of expired product with respect to respondent type as well total aggregated scores. Primarily descriptive nature was used to analyze the obtained data for present study. Responses to each of the survey questions were summarized by respondent type (gender) and for the overall sample. Box plot was also used to interpret the mean scores of respondents with respect to product preference for expiry date verification. 


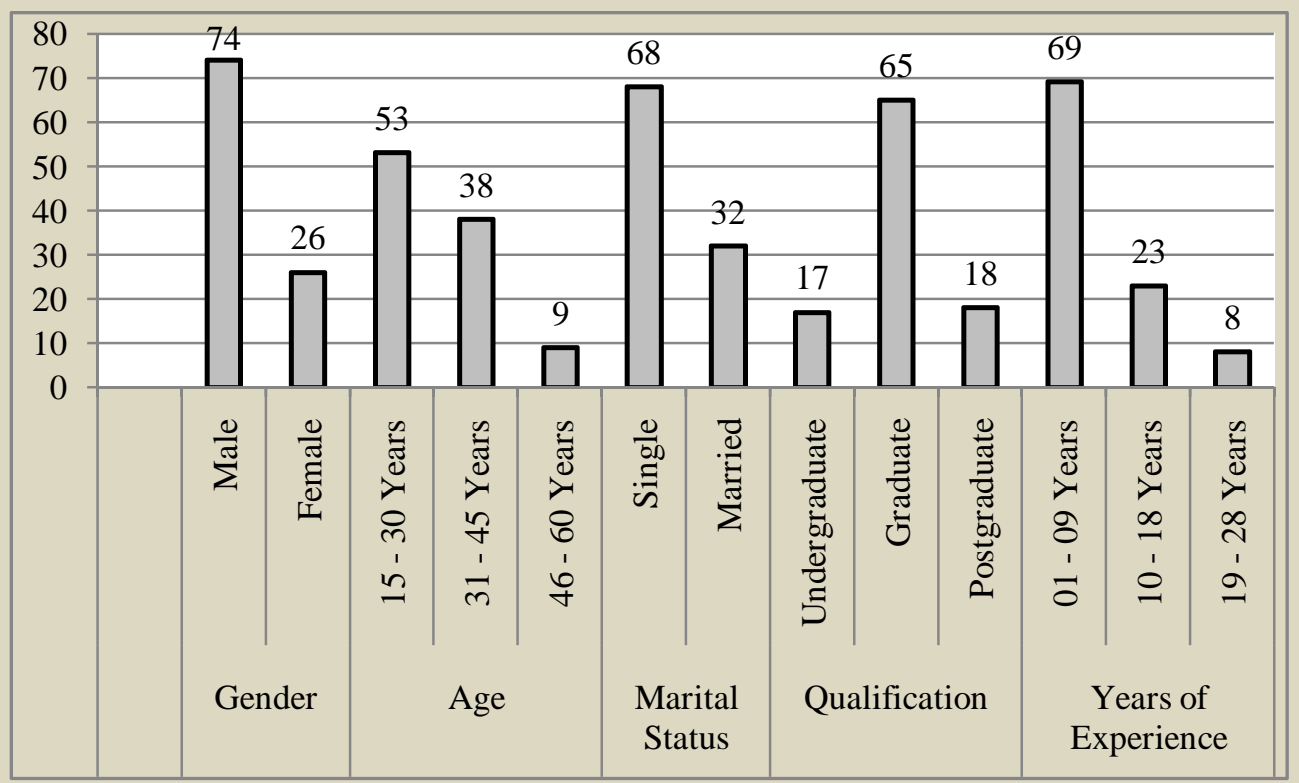

Fig. 1: Demographic characteristics of study participants.

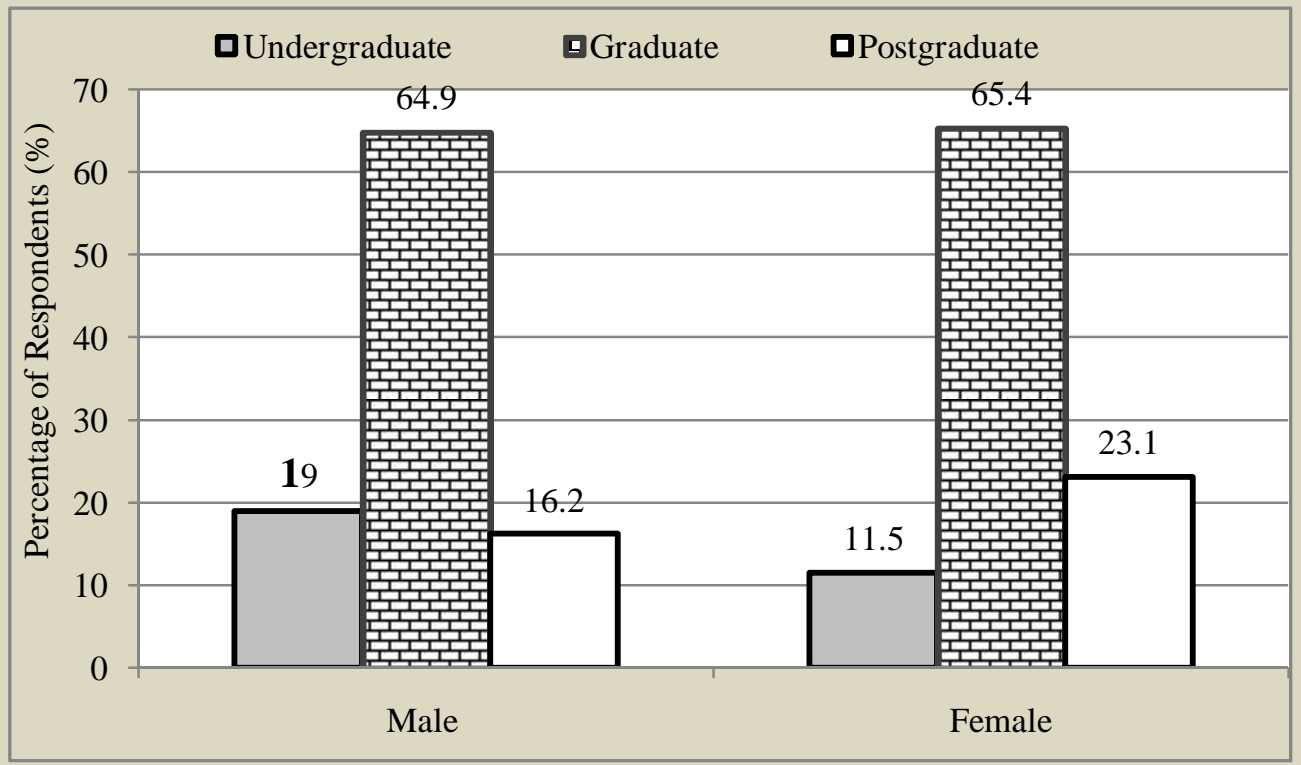

Fig. 2: Respondents' Distribution with respect to Qualification levels.

\section{RESULTS}

A total of 100 respondents completely filled the questionnaire, among them 74 were male and 26 were females. The age groups of respondents showed that 53 individual were in range of 15 to 30 years, 38 respondents were belongs to the age groups of 31 to 45 years while only 9 participants were of 46 to 60 age groups. Respondents which have single marital status were 68 whereas 32 were married. The qualification pattern showed that only 17 respondents were undergraduates, 65 respondents were graduated and 18 of them were postgraduate. The professional experience information showed that 69 of them have 1 to 9 years of experience, 23 individuals have 10 to 18 years of experience and only 8 of them have 19 to 28 years of experience (Figure 1).
Male participants were distributed with respect to qualification as $64.9 \%$ were graduated, $19 \%$ were undergraduates and $16.2 \%$ were postgraduate. In female participants $65.4 \%$ were graduated, $11.5 \%$ were undergraduates and $23.1 \%$ were postgraduates (Figure 2). However, gender based classification with respect to marital status showed that $64.9 \%$ male and $76.9 \%$ female were single whereas a low proportion of male i.e., $35.1 \%$ and of female i.e., $23.1 \%$ were married (Figure 3).

The mean scores of participants with respect to the level of awareness for pharmaceutical product expiry showed almost a similar consistency between different demographic variables as well as total mean scores (Table 2). More elaborately, with respect to gender the mean score of females respondents were $4.58 \pm 0.7$ and males have $4.64 \pm 0.77$. 
On the basis of marital status similar average was observed as single respondents have $4.59 \pm 0.8$ and married participants have $4.69 \pm 0.64$ awareness score. The qualification pattern showed the same practice as undergraduate respondents have mean score of $4.63 \pm 0.72$, graduated have $4.6 \pm 0.83$ and postgraduates have $4.67 \pm 0.49$. The total mean score of all respondents was $4.62 \pm 0.75$ (Table 2).

Table. 1: Average Scores of Respondents' Awareness for Expiry of Pharmaceutical Products with respect to demographic differentiation .

\begin{tabular}{llcc}
\hline \multirow{2}{*}{ Demographic Variables } & \multicolumn{2}{c}{ Scores } \\
\cline { 3 - 4 } Gender & Female & Mean & SD \\
& Male & 4.58 & 0.7 \\
& Single & 4.64 & 0.77 \\
\hline Marital Status & Married & 4.59 & 0.8 \\
& Under graduate & 4.69 & 0.64 \\
\hline Qualification & Graduate & 4.63 & 0.72 \\
& Postgraduate & 4.6 & 0.83 \\
& All Respondents & 4.67 & 0.49 \\
\hline Total Mean Score & & 4.62 & 0.75 \\
\hline
\end{tabular}

Respondents' perception regarding the product expiry was also assessed in which $6.8 \%$ male and $7.7 \%$ females were non-aware about the pharmaceutical product expiry concept. However, a big proportion of male $(93.2 \%)$ and females $(92.3 \%)$ were fully aware about this concept. A larger count of respondents from male $(87.8 \%)$ and females $(96.2 \%)$ were reported that they were not in favor to used expired products whereas, few respondents i.e., male $(12.2 \%)$ and females $(3.8 \%)$ were in disagreement to this subject. In addition to this, a larger group of respondents i.e., male $(89.2 \%)$ and female $(96.2 \%)$ reported the sever harmful effects of expired products, while a lowest total of $10.8 \%$ in male and $3.8 \%$ of females reported that the expired products has mild harmful effects (Figure 4).

Furthermore, the perceptions of respondents were taken regarding the expired product utilization or its discard after expiry date. Response showed that $20.3 \%$ male and $15.4 \%$ female were in view to utilize the expired products.
However, $79.7 \%$ male and $84.6 \%$ females were in agreement of its non-utilization. Similarly, $87.8 \%$ male and $76.9 \%$ females are in strong favor to discard the expired products while $12.2 \%$ male and $23.1 \%$ were against to this practice.

The main justification of this product was validated after taken the perception regarding its harmful impact on human health, according to which $83.8 \%$ male and all females of study were in strong favor for its harmful cause and consequences (Figure 5).

Respondent's knowledge and practice were also validated by taken their feedback on the purchase of expire products or product expiry verification before its purchase. Total of $84.2 \%$ male respondents and $73.1 \%$ females verified the product expiry before its purchase while a least proportion of $17.6 \%$ male and $26.9 \%$ female did not do this practice. Moreover, the perception and awareness regarding the purchase of short and long expiry product was also verified.

Least response was received from male i.e., $21.6 \%$ who purchase short expiry products, as well as similar in female i.e., $7.7 \%$. Contrary to this, $78.4 \%$ male prefer the long expiry products and $92.3 \%$ female also have had the similar practice (Figure 6).

Finally, the product preference with respect to expiry of pharmaceuticals and other were also taken which showed a highest response was achieved in the medicines i.e., $40.5 \%$ male and $46.2 \%$ females were in full agreement to expiry verification. Secondly, $18.9 \%$ male and $19.2 \%$ females prefers the expiry date verification on food items only, whereas, $10.9 \%$ male and $7.7 \%$ female used this practice on purchasing the cosmetic items only. Importantly, $29.7 \%$ male and $26.9 \%$ females were in opinion to have expiry verification in all these products (Figure 7). With respect to the product preference total mean score of participants were studied, it was observed that consistent mean score of around 4 to 5 units in all of these products with no reference of cosmetics (Figure 8).

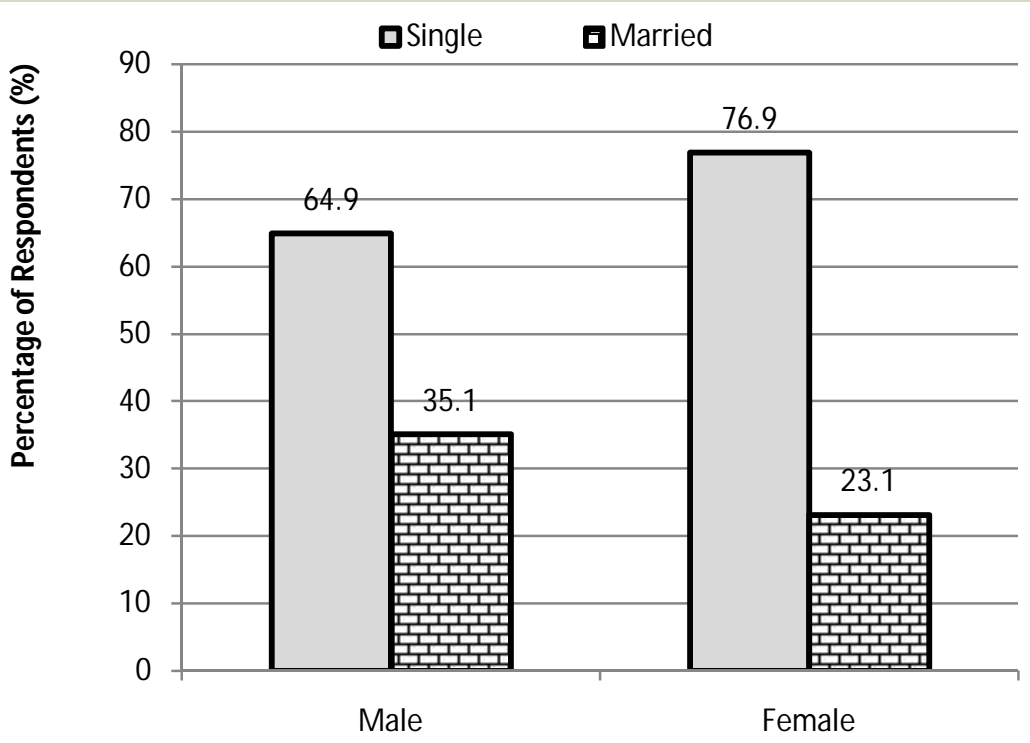

Fig. 3: Respondents' Distribution with respect to Marital Status. 


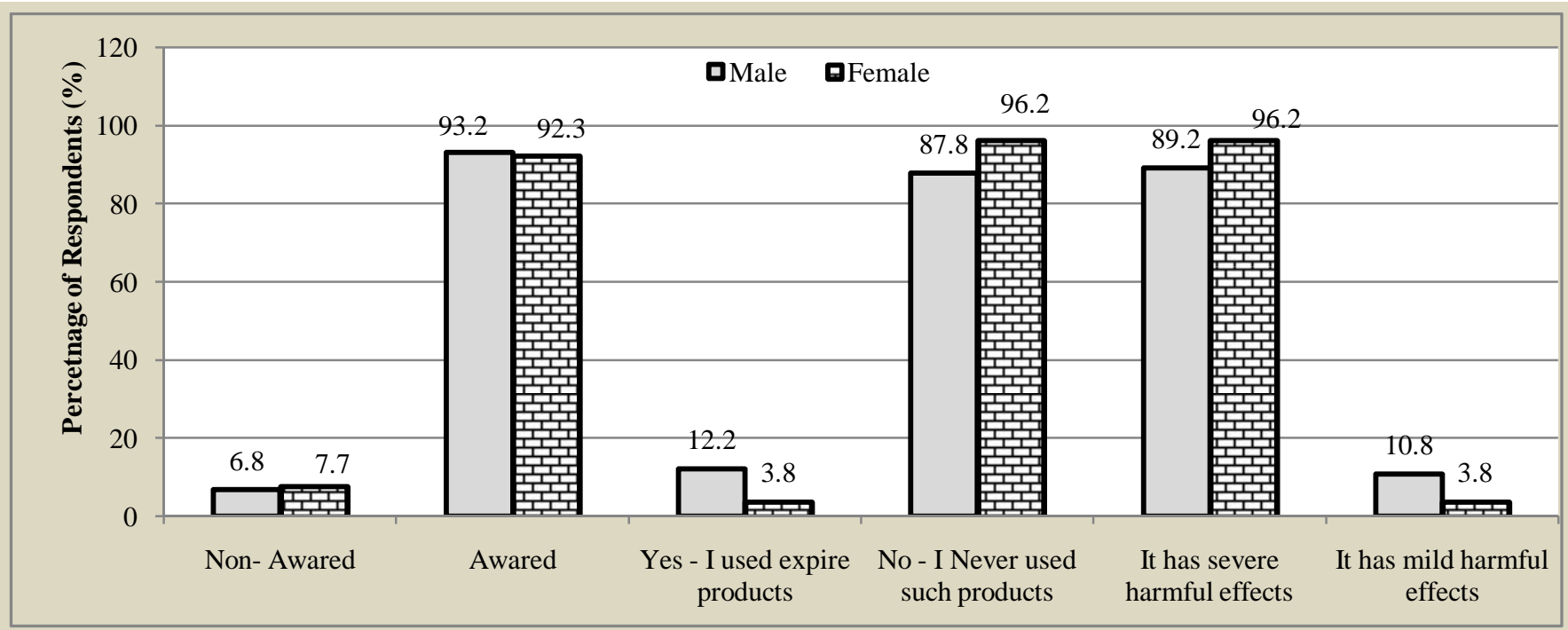

Fig. 4: Respondents perception about pharmaceutical products' expiry.

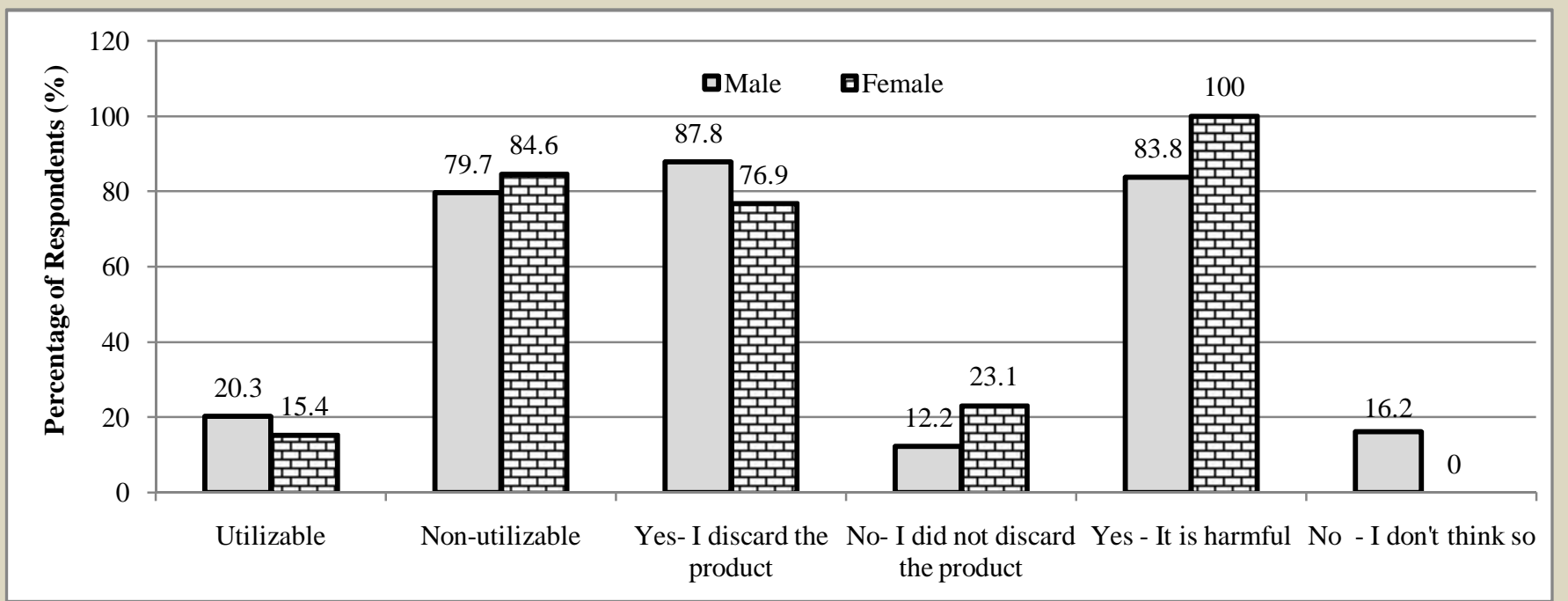

Fig. 5: Perceptions of respondents about the expired product utilization or its discard after expiry date.

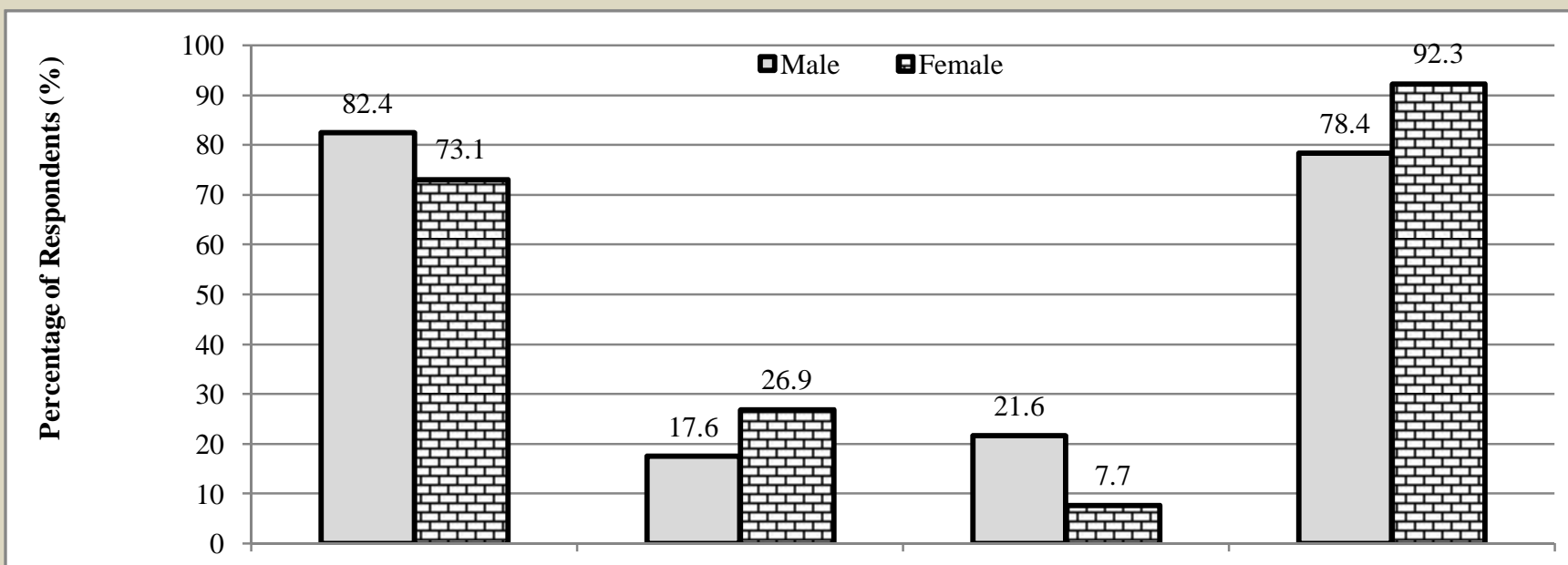

Yes - I check before No - I did not check before Buy short expiry product $\quad$ Buy long expiry product purchased purchase 


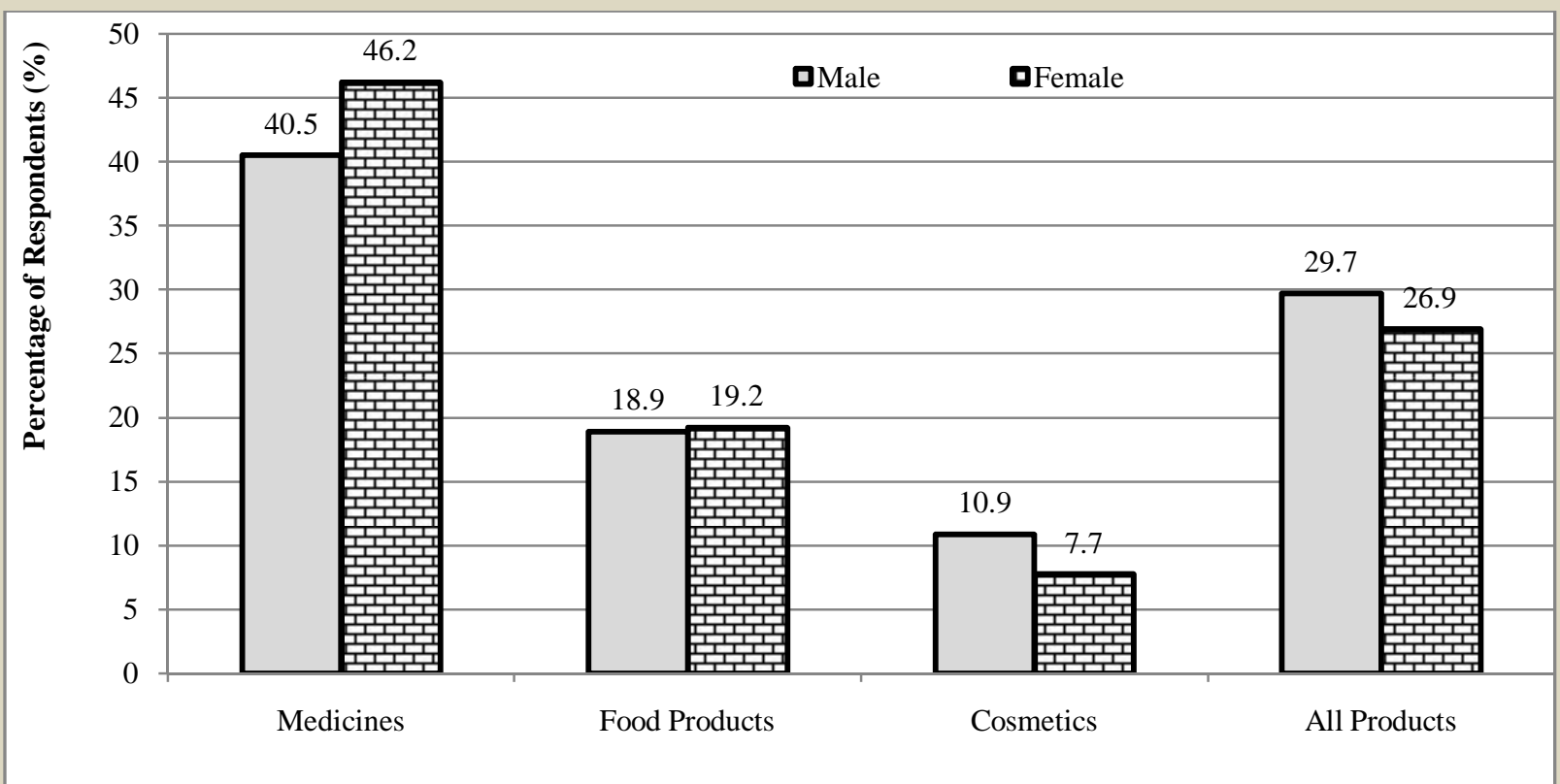

Fig. 7: Perception of respondents about Product Preference for expiry date.

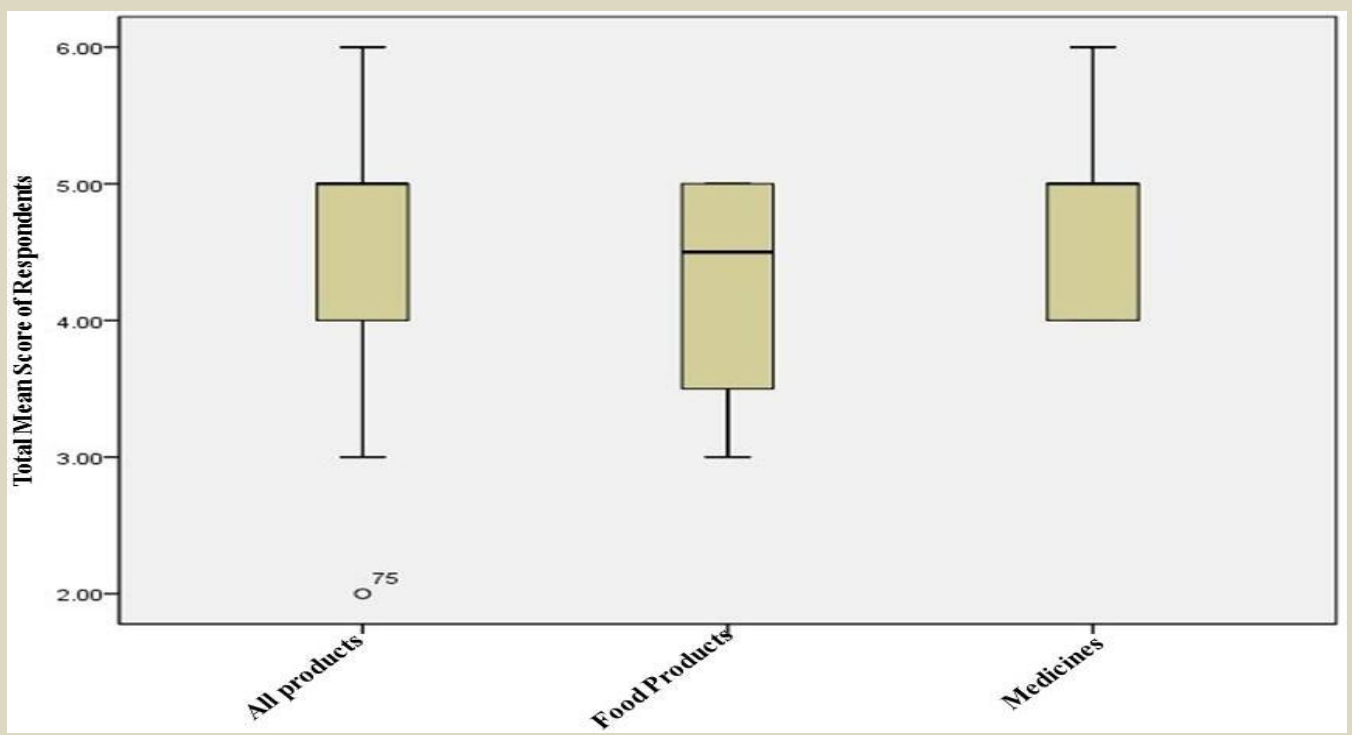

Fig. 8: Box Plot showing Product Preference with respect to participants mean score for expiry date awareness.

\section{DISCUSSION}

Pakistan, contributing the largest part in South Asian population, is facing a big challenge of illiteracy, and lack of scientific knowledge even in graduates. But from last few decades, a paradigm shift has been observed as different academic agencies and NGO's (non-government organization) are thriving hard to reduce this challenge. Due to this fact, the national strength for realizing the scientific needs and requirements are underdevelopment as vast groups of researches decodes the importance of awareness especially in the field of health and education (Alam et al., 2008; Shaikh and Hatcher, 2005). Moreover, little attention has been observed in educating people regarding the description of pharmaceuticals with reference to prescribed usage and discard (Janjua et al., 2006; Ahmed et al., 2013). Karachi is the largest metropolitan city of Pakistan as it represents multi-ethnic groups of Pakistani population. Therefore, the objective of present work was set accordingly to evaluate the general public awareness regarding the pharmaceutical product expiration. Our results showed a satisfactorily responses from the enrolled participants in present work. Additionally demographic characteristics of all respondents comprise a balanced spectrum in term of gender, age, marital status, qualification with experience of years. After stratification of demographic variables, the mean scores of respondents represent very least deviation in all the selected variables. On an account of average scores of respondent's awareness about expiration of pharmaceuticals, we observed awareness in grey area of highest level of this subject. 
This strengthened the concept of present effort that the majority knows about the product usage and expiry date but a consistent effort is still a need of next decades.

Beside this, an equal balanced in the percent frequency of both male and female respondents in relation with qualification status and marital status was noticed. Our results also suggested that highest frequencies of respondents have preliminary knowledge regarding the product expiration term as well as its non-utilization. After such interval, severe harmful impacts towards human health have been observed (Shah et al., 2011; Swaroop and Varun, 2011).

Earlier, it was reported that the assessment of awareness level is always a preferable marker to judge the community's knowledge, attitude and practices regarding the safe utilization of drugs, which generally represents public decision to seek the healthcare facilities, and use of medicines, and finally the success of treatments (Al-Gedadi et al., 2008; Shah et al., 2011). Furthermore, efficacy of medicines is very significant term because it defines the tendency of any medicine to produce the required effect.

The greater the efficacy, the more effective results the drug produced (Bajaj et al., 2012). On contrary, expired medicines may not relate with this above concept as because of reduced efficacy, inadequate treatment were observed which eventually lead to delay or increased in treatment mode, with loss of productivity (Swaroop and Varun, 2011).

In the same manner when treatment is consider, word 'medicine' always reflects as one of the most important entity which is necessarily required to treat ailments (Abdo-Rabbo et al., 2009). In this manner, the data for the perspective of respondents about the verification of pharmaceutical product showed good numbers of participants have awareness of checking the expiry of drug before purchase, and they preferred the purchase of long expiry date products.

This indicates that a good awareness level was observed in our urban population. Interestingly, the use of prescribed medicines is suitable to treat the diseases, however, if not taken appropriately, then it will cause harm instead of therapeutic effects (Swaroop and Varun, 2011; Kona et al., 2014). Despite of the benefits, previous evidences described the adverse reactions of medicines which were very common if a consumer not checked the date of expiry or buy the short or expired date products (Ekedahl, 2006).

Interestingly, after these findings the respondent's preferences of product with respect to observation of expiry were also taken. These findings reveal that a highest quantity of respondents have practiced to checked the expiration date specifically on medicines whereas, very least of them practiced the check of same date on food and cosmetic products. More importantly at the time of purchase of cosmetics and food product, the proper check of expiry date is always recommended as date of expiration is an indicative tool to measure the safety and nutritional quality. In case of the consumption of expired food, the prevalence of food-borne diseases also enhances (Sanlier, 2009).
Similarly, the use of cosmetics after their expiration may result in severs type of dermatological infection or may be a potent cause for the deformation of skin tissues (Nigam, 2009).

\section{CONCLUSION}

On the basis of these findings, it was concluded that the urban population of Karachi have sound knowledge on the expiry of pharmaceutical products as well as on its utilization. However, there is still space to educate the peoples regarding the same subject as this concept is expected to be still novel for the rest of population.

\section{LIMITATION OF STUDY}

This study has few limitations, which includes lack of research and survey studies in the concerned field, so difficult to generalize the findings in Pakistan. Language difference while dealing with the respondents was a major barrier while the respondents were free to give their response or regret; hence, output's reliability was not assured generally. Time duration and lack of funds directly reduces the survey sampling as well as further extension of this work.

\section{REFERENCES}

Persson M, Sabelström E, Gunnarsson Bo. Handling of unused prescription drugs-knowledge, behaviour and attitude among Swedish people. Environ. Intl. 2009; 35: 771-774.

Ali SE, Ibrahim MIM, Palaian S. Medication storage and selfmedication behaviour amongst female students in Malaysia. Pharm Pract, 2010; 8: 226-232.

Shah AP, Parmar SA, Ramkishan A, and Mehta AA. Knowledge, attitude and practice (KAP) survey regarding the safe use of medicines in rural area of Gujarat. Adv. Trop. Med. Pub. Health Int. 2011; 1: 66-70.

Al-Gedadi NA, Hassali MA, Shafie AA. A pilot survey on perceptions and knowledge of generic medicines among consumers in Penang, Malaysia. Pharm Pract. 2008; 6, 93-97.

Lyon RC, Taylor JS, Porter DA, Prasanna HR, Hussain AS. Stability profiles of drug products extended beyond labeled expiration dates. J Pharm. Sci. 2006; 95: 1549-1560.

Khan SR, Kona R, Faustino PJ, Gupta A, Taylor JS, Porter DA, Khan M. United States food and drug administration and department of defense shelf-life extension program of pharmaceutical products: progress and promise, J Pharm. Sci. 2014; 10: 1331-13366.

Swaroop AP, and Varun, D. 2011. A glimpse on expiry date of pharmaceutical dosage forms. Pharmanest.

Abdo-Rabbo A, Al-Ansari M, Gunn BC, Suleiman BJ, The use of medicines in Oman: public knowledge, attitudes and practices. Sultan Qaboos Univ. Med J. 2009; 9: 124-31.

Singh S. Drug stability testing and shelf-life determination according to international guidelines. Pharm. Technol. 1999; 23: 68-88.

Bajaj S, Singla D, Sakhuja, N. Stability testing of pharmaceutical products. J Apld. Pharm. Sci. 2012; 2: 129-138.

Carstensen JT. 2000. Drug stability, principles and practices, Marcel Dekker, New York.

Asti, Lindsey, Jones, R. Bridge J.A. Acetaminophen and expired medication storage in homes with young children. J Clinic Toxicol. 2012; 2 : 5 .

Alam, MZ, Aman R, Hafizullah, M. Patient awareness survey in A tertiary care hospital. JPMI. 2008; 22: 266-269.

Shaikh BT, Hatcher, J. Health seeking behaviour and health service utilization in Pakistan: challenging the policy makers. J Public Health (Oxf). 2005; 2: 49-54. 
Janjua NZ, Hutin, YJ, Akhtar, S, Ahmad, K. Population beliefs about the efficacy of injections in Pakistan's Sindh province. Public Health. 2006; 120: 824-833.

Ahmed A, Mushtaq N, Tariq M, Durrani M, Akhtar, S, Arif, M, Y, Ghazala. Disposal practices of unused and expired pharmaceuticals in Karachi and their impact on health and environment. JUMDC. 2013; 4, $42-48$.

Ekedahl ABE. Reasons why medicines are returned to Swedish pharmacies unused. Pharm. World Sci. 2006; 28: 352-358.

Sanlier N. The knowledge and practice of food safety by young and adult consumers. Food Control. 2009; 20: 538-542.
Nigam, PK. Adverse reactions to cosmetics and methods of testing. Indian J Dermatol Venereol Leprol. 2009; 75: 10-9.

\section{How to cite this article:}

Rafiq K., Azmi M. B., Saify Z. S., Khan W. U., Ashraf I., Rahim N Kumar G., Baloch N. Assessment of Knowledge and Practice on Consumption of Expired Pharmaceuticals: A Cross-Sectional Study at Karachi, Pakistan. J App Pharm Sci, 2015; 5 (04): 040-047. 